\section{TREATMENT OF MALIGNANT CHOLERA.}

\section{To the Editor of The LANCET.}

Sir, - After the numerous and some ably written articles on the treatment of cholera in your valuable journal, it may appear tedious and presumptuous in me to offer an opinion on the subject, and did I not feel that I was doing an act of justice to the medical profession and to the public, I should have remained silent. Knowing your liberality and desire to promote any information that might be useful, I am induced to draw up a plan of treatment which has been very successful in those cases which have fallen under my care, having reported to the Board of Health upwards of 160 cases, in consequence of my filling the situation of surgeon, \&c. to the poor of St. Jobn's, Southwark, the major part of which cases have been of the very worst character.

In the eariy commencement of the disease, and during the three first weeks, I adopted the different plans laid down by medical authors, which plans proved very unsuccessful ; almost every case died. I was then induced to try a plan of treatment of my own, which plan proved successful. When called to a case with all the bad symptoms of cholera, viz., vomiting and purging of rice-coloured fluids, cramps in the arms and legs, at times in the region of the stomach and diaphragm, coldness of the whole body, great prostration of strength, sunken eyes, \&c., the pulse scarcely to be felt at the wrist, the fingers and toes drawn and contracted, and aitended with great thirst, I commenced with giving, immediately, two grains of the extract of belladonna, washed down in one drachm of the tincture of ginger, with three drachms of water, and r peated in ten minutes if the cramps and sickness continued.' Mustard poultices to the whole of the chest, abdomen, and spine, were kept on till the patient complained of great pain and uneasiness, and the pulse increased in volume, which generally took place in about fifteen minutes. I then commenced with giving three grains of calomel with half a grain of powdered opium, every hour for four hours, keeping at the same time the patient from taking any hind of fluid, save and except a teaspoonful of toast-and-water occasionally to wet his or her lips. This treatment, $\mathrm{I}$ am happy to say, has proved itself truly satisfactory. Taking the average of cases, I have been able to save eight out of twelve, where all the bad symptoms of cholera have prevailed. After the lapse of six or eight hours, if the patient has not had an evacuaion, nor passed water, which is one of the characteristic symptoms of cholera, I gave half a drachm of powdered rhubarb with a ittle conf. aromat. every three or four hours, with a mild emollient glyster; this generally produced the desired effect, and my patients did well, leaving great debility, \&c. At times, after the first stages of the disease, consecutive fever followed ; this I have found yield best to carbon. sodæ, in doses of five grains every three or four hours, with mild diluents, veal, or chicken broth, with rice boiled in it; then avoiding all stimulants until all inflammation or ever have subsided. I am, \&c.

Wm. Mrskin, Surgeon, \&c.

8, Broad-street, Horselydown, May 4th, 1832.

CASE OF

\section{MALIGNANT CHOLERA.}

RELATED BY THE PATIENT.

\section{To the Editor of 'The LanceT.}

Si $R,-$ Having experienced in my own person a violent attack of the prevailing epidemic, I am induced to give publicity to the case in consequence of the very unfavourable auspices under which it was treated. Previously to detailing the particulars of the case, I ought to say that to the unre. mitting kindness and attentiou of the medical attendants, my restoration to bealth is entirely due.

I am 22 years of age, and a pupil of St. Bartholomew's Hospital. About three months since, having wounded my hand in dissecting, so much irritative fever ensued as greatly impaired my general health. In this weakened state of constitution, when the cholera made its appearance in this city I became much engaged with MIr. Kiernan and M. Halmagrand in prosecuting their investigations into its nature, assisting in many post-mortem inspections, as well as several times witnessing the various precursory symptoms of the disorder. On Thursdar, the 12th of April, I attended as Mr. Kiernan's assistant to inspect a case of malignant cholera which had proved fatal, in New Street, Cloth Fair, and on the morning of the following Saturday I was present at an examination in the same house, of a woman who had struggled with the disease for several days, and had died in the last or typhoid stage of the complaint. During the afternoon I felt a degree of lassitude, and had two loose and griping evacuations with a painful sensation, as i/ my stomach and intestines were spasmodi cally affected, although I had carefully ab. 